\title{
A Crossover Design for Comparative Efficacy: A 28 Days- Randomized Clinical Trial of Cognitive Behavioral Therapy for Chronic Pain of Degenerative Conditions
}

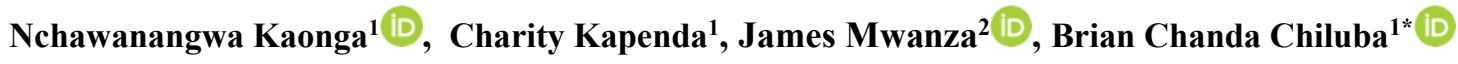 \\ ${ }^{1}$ The University of Zambia, School of Health Sciences, Department of Physiotherapy, ${ }^{2}$ The University of Zambia, School of Medicine, \\ Department of Psychiatry
}

*Corresponding author: brian.chiluba@unza.zm

To cite: Nchawanangwa K, Kapenda C, Mwanza J, Chiluba BC,. A Crossover Design for Comparative Efficacy: A 28 Days-Randomized Clinical Trial of Cognitive Behavioral Therapy for Chronic Pain of Degenerative Conditions. JPRM 2021, 3(2): 42-55. doi: 10.21617/jprm2021.329

In physiotherapy practice, a number of patients are known to suffer from chronic pain which results in reduced activity levels, interference in sleep, enjoyment of life, mood, and relations with others. Cognitive behavioral therapy, in this aspect will provide a holistic approach to the available treatment. Cognitive behavioral therapy has also been shown to target cognitive distortions such as pain catastrophizing, fear avoidance, overgeneralizing and others, all the while improving physical health, activity levels and quality of life. This study assessed the efficacy of cognitive behavioral therapy in the management of patients with chronic pain at the University Teaching Hospitals in Zambia. A randomized clinical trial utilizing a crossover design was utilized for the study. A random sample of 32 participants was used in the study after fulfilling the study criteria. Data was analyzed using ANCOVA with alpha of 0.05 . The study recorded small effects in the reduction of pain intensity in both phase one and two. In phase one, it also recorded small effects in general work interference, sleep interference and enjoyment of life interference but recorded medium effects in normal work interference and mood interference. Phase two of the study recorded small effect size in reduction of general work interference, normal work interference, mood interference, relations with others interference, sleep interference and Enjoyment of Life interference. Cognitive behavioral therapy ensures the management of chronic pain addresses areas in which an individual is affected by pain and which in turn exacerbate the chronic pain. Physiotherapy provides a more holistic approach when used in conjunction with cognitive behavioral therapy.

Keywords: Cognitive behavioral therapy, chronic pain, physiotherapy, crossover design 


\section{INTRODUCTION}

Pain can be described as acute or chronic with chronic being pain that persists for at least three months and acute being pain that persists for less than three months. Chronic pain is associated with psychological and social burden. Chronic pain is said to be treated by Cognitive Behavioral Therapy (CBT) [1].

CBT is a psychotherapeutic treatment concept that comprises the elements of behavioral therapy and cognitive Therapy. It is mainly based on operant conditioning and elements from cognitive therapy. It is an umbrella term for a broad variety of interventions whose aims are to alter maladaptive thoughts, feelings and behavior as well as dysfunctional sensory phenomena, and thereby the experience of pain [2]. CBT was invented by the psychiatrist Aaron Beck in the 1960s when he discovered what he termed as automatic thoughts. According to Beck, these thoughts were the key to the client or patient understanding and overcoming their difficulties. Due to the emphasis that was placed on thinking, Beck termed it as Cognitive Therapy. Cognition can be defined as a verbal or pictorial event in an individual's stream of consciousness. Cognition is based on the individual's attitudes or assumptions that are developed from past events. However, the therapy employs behavioral techniques as well hence it is now known as CBT $[3,4]$

Melzack and Wall, gate control theory emphasizes the importance of cognitive, affective and sensory influence on chronic pain. The cognitive aspect of chronic pain treatment by Wilbert Fordyce's applications of learning theory and operant behavioral principles to pain involves the application of behaviors [5]. This is because it helps in the understanding and treatment of chronic pain. Wilbert Fordyce's application resulted in the expansion of chronic pain treatments to include Behavioral treatments that aimed to decrease patients' pain behaviors such as limping and increase "well" behaviors such as participation in customary activities [6].

An individual's belief in his ability to perform a behavior or achieve a desired outcome $[7,8]$, determines the thoughts, feelings, and behaviors in stressful situations and affects one's ability to cope successfully when confronted with challenges [9]. Measures of pain-related selfefficacy assess patients' perceived ability to control pain symptoms and function in spite of pain $[10,7]$. Pain related avoidance, a factor for patients with persistent pain and a resiliency factor for children, adolescents, and adults with chronic pain, predicts functional pain outcomes. In samples of adults and children with chronic headaches, pain-related self-efficacy is associated with less disability, better functioning, and fewer depressive symptoms [11,12]. That is, patients with higher levels of pain have lower self-efficacy, which is associated with increased difficulty performing valued activities. On the other hand, in a longitudinal study of patients with chronic back pain, improvements in self-efficacy partially mediated the relationship between changes in pain and disability with improvements in pain leading to increased self-efficacy which in turn resulted in less pain-related disability [13]. CBT as a nonpharmacologic treatment for chronic pain, targets pain-related self-efficacy as a process variable with the aim of improving self-efficacy for making such adjustments and living with pain. Self-efficacy has been shown to be a key mechanism of change in CBT for chronic pain $[14,15,16]$.

The efficacy of CBT for individuals with chronic pain has been evaluated in randomized controlled trials (RCTs) for over three decades, primarily in samples of adults with chronic back pain, headaches, orofacial pain, or arthritis related pain [6].

This study set out to assessed the efficacy of cognitive behavioral therapy in the management of patients with chronic pain at the University Teaching Hospitals (UTH) in Zambia.

\section{METHODS AND MATERIALS}

This study was a single-center, randomized, single subject blind, 2-way crossover study design. Its enrolled participants who had a degenerative condition being treated at the UTH, in the department of physiotherapy. The study consisted of two separate intervention periods each lasting 2 weeks. The objective of the study was to assess the efficacy of CBT in the management of patients with chronic pain at the UTH, Department of Physiotherapy.

\section{Primary Endpoints}

The co-primary endpoints were the change from baseline in mean pain intensity at 2 weeks. Pain intensity was measured at the beginning for at least 5 minutes. Pain intensity was measured with a brief pain tool time at the beginning of the study and a mean of three measurements was calculated. The last participant completed the sessions on 19/07/2019 and the pain intensity was measured again on 23/07/2019. Secondary endpoints

The study adopted six (6) secondary 

Clinical Trial of Cognitive Behavioral Therapy for Chronic Pain of Degenerative Conditions.

endpoints from the questionnaire. Each endpoint was analyzed and discussed individually under a heading. The secondary endpoints are based on the patients' response to chronic pain.

\section{Study setting and Population}

This study was conducted at the University Teaching Hospitals (UTH) in Zambia, The UTH is the biggest hospital in Zambia and is located in the capital city, Lusaka. It is approximately $4 \mathrm{~km}$ east of the city centre. UTH provides a full range of primary, secondary and tertiary health and medical services on both an inpatient and outpatient basis. All patients aged 18 to 80 years who have experienced pain that has persisted for three months or more during the study period were enrolled. Any Patients that were not mentally sound, had neuropathies or radiculopathies and pregnant women were excluded.

A simple random sampling method was used in the study. The researcher selected participants who by virtual of their symptoms fell into the inclusion criteria. This sampling method was chosen because the study and follow-ups had to be done within the shortest period of time.

With the use of an online calculator, a study group design of two independent study groups and a continuous primary endpoint. Statistical parameters were set as follows; alpha of 0.05 , power of $80 \%$, anticipated mean 1 of $39+/-1$, anticipated mean 2 of 40 and enrollment ratio of 1 . The sample size of 32 participants was concluded with the control group having 16 participants and the intervention group having 16 participants. The calculator site used was https://clincalc.com/stats/samplesize.aspx

\section{Study procedure}

The study was divided into two phases (Phase one and two) in which patients were randomized into separate groups. One group received Physiotherapy only for seven sessions while the other group received a combination of Physiotherapy and CBT.

\section{Phase one}

For phase one, the intervention group received seven sessions. Session one included an interview and assessment using the questionnaire. The participant's pain was assessed at the beginning of the phase and the participant was familiarized with treatment approach. In session two the participant was given feedback from the assessment and treatment goals were planned. Session three was about exercise and pacing. The participant was taught the importance of physical activities done with a thoughtful approach. The participant was then given an exercise regimen or prescription. In session four, the participant was taught relaxation techniques such as progressive muscle relaxation, stretch based relaxation and deep breathing. For session five the participant was asked to identify a pleasant activity. The identified activity was then implemented and feedback was obtained. For session six, was taught cognitive coping in which they were made to understand how automatic negative thoughts impacted their pain experience. The participants were then told to monitor their automatic thoughts and challenge the negative thoughts. Session seven looked at the discharge of the participant from the intervention group. The participant was taught how to handle any pain flare-ups when they happened. Their CBT skills were also reviewed. The pain intensity and pain effects on physical activities (general work and normal activities), mood, sleep, relations with other people and enjoyment of life was re-evaluated.

The control group on the other hand received the conventional physiotherapy treatment. The participants were interviewed using the questionnaire and their pain and its effects were evaluated. They then underwent management using electrotherapy, heat and exercises. At the end of the phase, the participants' pain and its effects on physical activities (general work and normal activities), mood, sleep, relations with other people and enjoyment of life were re-evaluated.

\section{Phase two}

Phase two begun with a crossover of group members. The participants from the intervention group crossed over to the control group while the participants from the control group crossed over to the intervention group. Each participant's pain was evaluated at the beginning of the phase.

The intervention group had seven sessions done before the participants were reevaluated. Session one included an interview and assessment using the questionnaire. The participant's pain was assessed at the beginning of the phase and the participant was familiarized with treatment approach. In session two the participant was given feedback from the assessment and treatment goals were planned. Session three was about exercise and pacing. The participant was taught the importance of physical activities done with a thoughtful approach. The participant was then given an exercise regimen or prescription. In session four, the participant was taught relaxation techniques such as progressive muscle relaxation, stretch based relaxation and deep breathing. For session five the participant was asked to identify a pleasant activity. The identified activity was then implemented and 
feedback was obtained. For session six, was taught cognitive coping in which they were made to understand how automatic negative thoughts impacted their pain experience. The participants were then told to monitor their automatic thoughts and challenge the negative thoughts. Session seven looked at the discharge of the participant from the intervention group. The participant was taught how to handle any pain flare-ups when they happened. Their CBT skills were also reviewed. The participants' pain intensity and pain effects on physical activities, mood, sleep, relations with other people and enjoyment of life were reevaluated.

In the control group, conventional physiotherapy treatment was used. The participants were interviewed using the questionnaire and their pain and its effects were evaluated. They then underwent management using electrotherapy, heat and exercises. At the end of the phase, the participants' pain and its effects on physical activities, mood, sleep, relations with other people and enjoyment of life were re-evaluated.

\section{Trial Procedures}

Patients received a comprehensive evaluation at baseline in order to determine if there was a lag effect differentiating interventions. Institutional review board/independent ethics committee approval was obtained at both sites and all participants gave written informed consent. The study was conducted in accordance with the tenets of the Declaration of Helsinki of 2013. No stipend was given for participation. There was no independent data and safety monitoring committee in this study as there are no safety issues that could have arisen for CBT intervention apart from psychosocial issues which were addressed by comprehensive provision of information using the information sheet. The study is registered at Pan African Clinical Trials Registry of the South African Medical Research Council with registration number PACTR201906866179350-

https://pactr.samrc.ac.za/TrialDisplay.aspx?TrialI $\mathrm{D}=8190$. A register that is a subscription member of WHO for clinical trials.

\section{Statistical Analysis}

All participants who received at least one treatment session of each intervention (Physiotherapy and Physiotherapy + CBT) and completed all seven study visits were included in the efficacy analysis. A sample size of 32 participants was needed to provide $80 \%$ power to detect a 1 del difference in pain dimensions. ANCOVA was used to compare the difference between participants receiving CBT and PT, with the collection of pain dimension at baseline, gender and age as covariates, and the treatment group as a factor. The test was performed with a significance level of 0.05 (two-sided). A differential first-order carry-over effect of $3 \%$ (i.e., $20 \%$ of effect of previous period would be maintained through a subsequent period) was assumed. Statistical analyses were carried out using SPSS version 25.

\section{RESULTS}

A total of 27 patients with chronic degenerative pain were recruited from the UTH. All of participants were above 18 years of age with the youngest being 25 years old and the oldest being 74 years old. Females made up $63.00 \%$ of the participants while males made up $37.00 \%$ of the participants. Of the 27 participants, 21 suffered from conditions of the lumbar region only, 2 from conditions of the cervical region only, 1 from conditions of the thoracolumbar region only, 1 from conditions of both the cervical and lumbar region and 2 from conditions of the knee. The questions were asked in the language that the participants were most comfortable with.

Chronic pain was seen to affect individuals over a variety of occupations and professions. The longest reported pain duration was 216 months (18 years) while the least reported pain duration was 3 months and the most prevalent was 24 months ( 2 years).

Pain killers were seen to be the most prevalent sort after treatment. Among the recruited participants, $59.30 \%$ relied on painkillers only, $7.40 \%$ relied on Physiotherapy only, $29.60 \%$ relied both on pain killers and Physiotherapy and only $3.70 \%$ had never sort any treatment prior to the study. The baseline characteristics are shown in table 1 . 
Table 1: Change from baseline in mean pain intensity scores at 2 weeks for randomized patients on CBT and PT treatments at UTH

\begin{tabular}{|c|c|c|c|}
\hline MEASURE & $\begin{array}{l}\text { CBT }(\mathrm{N}=16) \\
M E A N\end{array}$ & $\begin{array}{l}\text { CONTROL }(\mathrm{N}=11) \\
M E A N\end{array}$ & P-VALUE \\
\hline \multicolumn{4}{|c|}{ Phase one pain intensity } \\
\hline \multicolumn{4}{|l|}{ Baseline } \\
\hline -Present pain & 3.79 & 2.61 & 0.02 \\
\hline -Worst pain & 4.85 & 3.34 & \\
\hline -Least pain & 2.25 & 1.55 & \\
\hline \multicolumn{4}{|l|}{ Change at 2 weeks } \\
\hline -Present pain & 2.78 & 1.92 & \\
\hline -Worst pain & 3.77 & 3.00 & \\
\hline -Least pain & 1.66 & 1.15 & \\
\hline \multicolumn{4}{|c|}{ Phase two pain intensity } \\
\hline \multicolumn{4}{|l|}{ Baseline } \\
\hline -Present pain & 2.79 & 1.91 & 0.02 \\
\hline -Worst pain & 3.77 & 2.60 & \\
\hline -Least pain & 1.67 & 1.14 & \\
\hline \multicolumn{4}{|l|}{ Change at 2 weeks } \\
\hline -Present pain & 2.15 & 1.47 & \\
\hline -Worst pain & 3.31 & 2.28 & \\
\hline -Least pain & 1.14 & 0.79 & \\
\hline
\end{tabular}

\section{Phase one result}

Primary endpoint: Pain Intensity

During phase one, the intervention group showed a mean present pain intensity of 3.88 , a mean worst pain of 5.00 and a mean least pain of 1.44. On the other hand, the control group showed a mean present pain intensity of 5.91, a mean worst pain of 8.36 and a mean least pain of 4.82 .
In table 2, a one way between subjects ANCOVA was conducted to determine a statistically significant effect of treatment on pain intensity after controlling for initial intensity, present pain intensity (IPP) $\mathrm{F}(1,24)=2.58, p=0.09$, worst pain (WP) $\mathrm{F}(1,24)=4.99, p=0.04$, least pain (LP) $\mathrm{F}(1,24)=6.64, p=0.02$.

\section{Table 2: Tests of between-subject's effects of chronic pain patients with degenerative conditions at UTH}

\begin{tabular}{|c|c|c|c|c|c|c|c|}
\hline $\begin{array}{l}\text { Dependent } \\
\text { Variable }\end{array}$ & Source & $\begin{array}{l}\text { Type III } \\
\text { Sum of } \\
\text { Squares }\end{array}$ & Df & $\begin{array}{c}\text { Mean } \\
\text { Square }\end{array}$ & $F$ & Sig. & $\begin{array}{c}\text { Partial } \\
\text { Eta } \\
\text { Squared }\end{array}$ \\
\hline \multirow{5}{*}{$\begin{array}{l}\text { Present } \\
\text { Pain (post } \\
\text { treatment) }\end{array}$} & $\begin{array}{l}\text { Corrected } \\
\text { Model }\end{array}$ & $51.58^{\mathrm{a}}$ & 2.00 & 25.79 & 5.84 & 0.10 & 0.33 \\
\hline & Intercept & 16.58 & 1.00 & 16.58 & 3.75 & 0.07 & 0.14 \\
\hline & IPP & 24.60 & 1.00 & 24.60 & 5.57 & 0.03 & 0.19 \\
\hline & Treatment & 11.39 & 1.00 & 11.39 & 2.58 & 0.12 & 0.10 \\
\hline & Error & 106.06 & 24.00 & 4.42 & & & \\
\hline \multirow{5}{*}{$\begin{array}{l}\text { Worst Pain } \\
\text { (post } \\
\text { treatment) }\end{array}$} & $\begin{array}{l}\text { Corrected } \\
\text { Model }\end{array}$ & $111.58^{\mathrm{a}}$ & 2.00 & 55.79 & 8.44 & 0.00 & 0.41 \\
\hline & Intercept & 6.40 & 1.00 & 6.40 & 0.97 & 0.34 & 0.04 \\
\hline & IWP & 37.83 & 1.00 & 37.83 & 5.72 & 0.03 & 0.19 \\
\hline & Treatment & 33.02 & 1.00 & 33.02 & 4.99 & 0.04 & 0.17 \\
\hline & Error & 158.72 & 24.00 & 6.61 & & & \\
\hline \multirow{5}{*}{$\begin{array}{l}\text { Least Pain } \\
\text { (post } \\
\text { Treatment) }\end{array}$} & $\begin{array}{l}\text { Corrected } \\
\text { Model }\end{array}$ & $138.60^{a}$ & 2.00 & 69.30 & 17.42 & 0.00 & 0.59 \\
\hline & Intercept & 3.58 & 1.00 & 3.582 & 0.90 & 0.35 & 0.04 \\
\hline & ILP & 64.10 & 1.00 & 64.10 & 16.11 & 0.00 & 0.40 \\
\hline & Treatment & 26.40 & 1.00 & 26.40 & 6.64 & 0.02 & 0.22 \\
\hline & Error & 95.48 & 24.00 & 3.98 & & & \\
\hline
\end{tabular}




\section{Secondary Endpoints}

General Work

Results under general work showed a

CBT mean of 3.50, [F $(1,24)=4.94, p=0.04]$, a control mean of 5.73 and initial general work $[\mathrm{F}$ $(1,24)=7.16, p=0.01]$

\section{Mood}

Results under mood interference showed a CBT mean of 0.50, $[\mathrm{F}(1,24)=9.32, p=0.05]$ and a control mean of 3.64 , with initial mood interference $[\mathrm{F}(1,24)=14.03, p=0.01]$

Normal work

Results under 'normal work' interference showed a CBT mean of 2.94, [F $(1,24)=6.77, p$ $=0.02]$ and a control mean of 6.09 with initial normal work $[\mathrm{F}(1,24)=8.30, p=0.08]$

Relations with people
Results under 'relations with other people' interference showed a CBT mean of 0.56 , $[\mathrm{F}(1,24)=0.007, p=0.936]$ with a control mean of 3.64 and RWP $[\mathrm{F}(1,24)=170.90, p=0.00]$

Sleep

Results under sleep interference showed a CBT mean of 1.31, [F $(1,24)=3.80, p=0.06]$, a control mean of 3.91 and an initial sleep interference $[\mathrm{F}(1,24)=117.76, p=0.00]$

Enjoyment of Life

Results under 'enjoyment of life (EOL)' interference showed a CBT mean of 2.31, [F (1, $24)=2.99, p=0.10]$ with a control mean of 5.18 and an initial 'EOL' $[\mathrm{F}(1,24)=17.17, p=0.00]$. Details of the description of the secondary end points are shown in figure 1 with the ANCOVA results in table 3 .

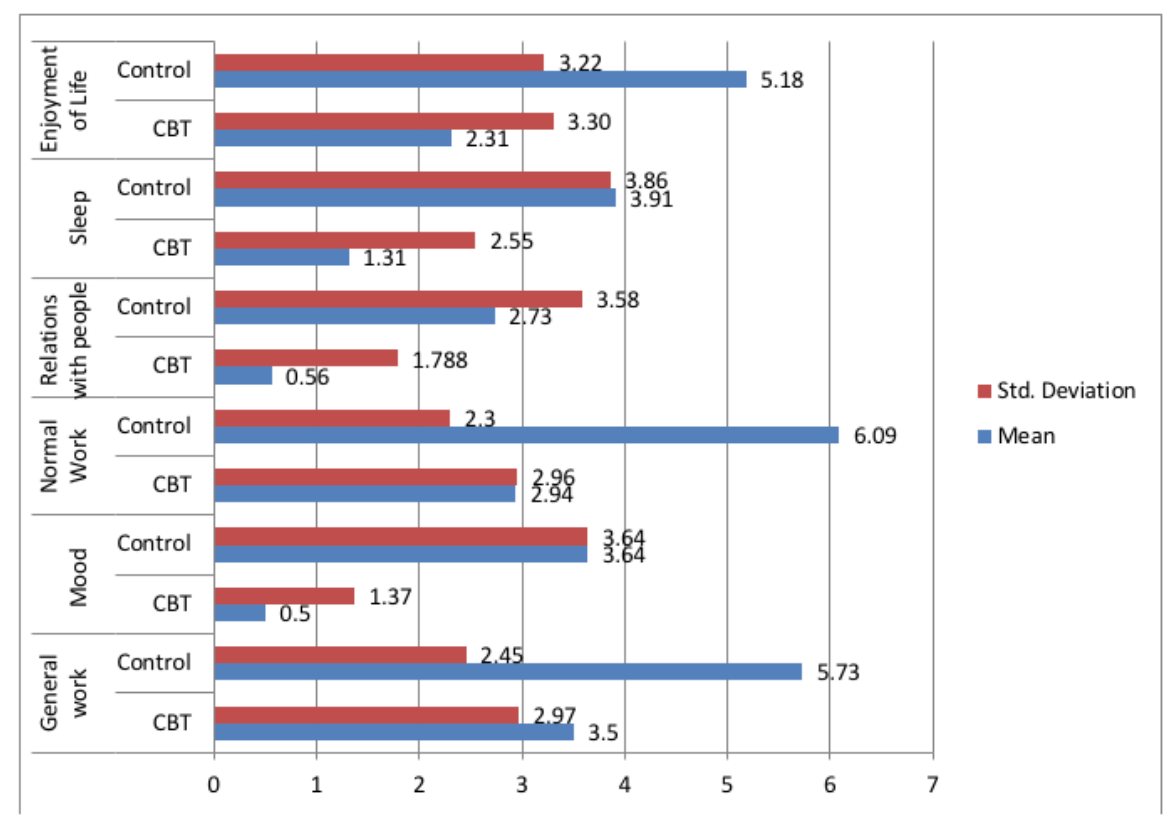

Figure 1: Descriptive Statistics of secondary outcomes of chronic pain patients with degenerative conditions at UTH 
Nchawanangwa K, Kapenda C, Mwanza J, Chiluba BC,. A Crossover Design for Comparative Efficacy: A 28 Days-Randomized Clinical Trial of Cognitive Behavioral Therapy for Chronic Pain of Degenerative Conditions.

Table 3: Tests of Between-Subject Effects of secondary outcomes of chronic pain patients with degenerative conditions

\begin{tabular}{|c|c|c|c|c|c|c|c|}
\hline Variable & Source & $\begin{array}{l}\text { Type III } \\
\text { Sum of } \\
\text { Squares }\end{array}$ & $d t$ & $\begin{array}{l}\text { Maan } \\
\text { Square }\end{array}$ & $F$ & Sig. & $\begin{array}{l}\text { Partal } \\
\text { Eta } \\
\text { Squared }\end{array}$ \\
\hline \multirow[t]{5}{*}{ General Work } & $\begin{array}{l}\text { Corrected } \\
\text { Model }\end{array}$ & $76.48^{\circ}$ & 2.00 & 38.24 & 6.20 & 0.01 & 0.34 \\
\hline & Tntercept & 25.66 & 1.00 & 25.66 & 4.16 & 0.05 & 0.15 \\
\hline & TGW & 44.15 & 1.00 & 44.15 & 7.16 & 0.01 & 0.23 \\
\hline & Treatment & 30.45 & 1.00 & 30.45 & 4.94 & 0.04 & 0.17 \\
\hline & Error & 148.04 & 24.00 & 6.17 & & & \\
\hline \multirow[t]{5}{*}{ Mood } & $\begin{array}{l}\text { Corrected } \\
\text { Model }\end{array}$ & $123.35^{2}$ & 2.00 & 61.68 & 14.61 & 0.00 & 0.55 \\
\hline & Intercept & 6.05 & 1.00 & 6.05 & 1.43 & 0.24 & 0.06 \\
\hline & $T M$ & 59.23 & 1.00 & 59.23 & 14.03 & 0.00 & 0.37 \\
\hline & Treatment & 39.34 & 1.00 & 39.34 & 9.32 & 0.01 & 0.28 \\
\hline & Error & 101.31 & 24.00 & 4.22 & & & \\
\hline \multirow[t]{5}{*}{ Normal Work } & $\begin{array}{l}\text { Corrected } \\
\text { Model }\end{array}$ & $112.07^{u}$ & 2.00 & 56.03 & 9.85 & 0.00 & 0.45 \\
\hline & Tntercept & 9.11 & 1.00 & 9.11 & 1.60 & 0.22 & 0.06 \\
\hline & $\mathbb{T N W}$ & 47.25 & 1.00 & 47.25 & 8.30 & 0.01 & 0.26 \\
\hline & Treatment & 38.55 & 1.00 & 38.55 & 6.77 & 0.02 & 0.22 \\
\hline & Error & 136.60 & 24.00 & 5.69 & & & \\
\hline \multirow[t]{5}{*}{ Relations With People } & $\begin{array}{l}\text { Corrected } \\
\text { Model }\end{array}$ & $184.98^{\circ}$ & 2.00 & 92.49 & 102.35 & 0.00 & 0.90 \\
\hline & Tntercept & 0.03 & 1.00 & 0.03 & 0.03 & 0.87 & 0.00 \\
\hline & TRWP & 154.43 & 1.00 & 154.43 & 170.90 & 0.00 & 0.68 \\
\hline & Treatment & 0.01 & 1.00 & 0.01 & 0.01 & 0.94 & 0.00 \\
\hline & Error & 21.69 & 24.00 & 0.90 & & & \\
\hline \multirow[t]{5}{*}{ Sleep } & $\begin{array}{l}\text { Corrected } \\
\text { Model }\end{array}$ & $161.71^{4}$ & 2.00 & 80.66 & 15.09 & 0.00 & 0.56 \\
\hline & Intercept & 0.38 & 1.00 & 0.38 & 0.07 & 0.79 & 0.00 \\
\hline & TS & 117.76 & 1.00 & 117.76 & 21.98 & 0.00 & 0.48 \\
\hline & Treatment & 20.35 & 1.00 & 20.35 & 3.60 & 0.06 & 0.14 \\
\hline & Error & 128.59 & 24.00 & 5.36 & & & \\
\hline \multirow[t]{5}{*}{ Enjoyment of Life } & $\begin{array}{l}\text { Corrected } \\
\text { Model }\end{array}$ & $165.05^{4}$ & 2.00 & 82.53 & 12.72 & 0.00 & 0.52 \\
\hline & Tntercept & 0.49 & 1.00 & 0.49 & 0.08 & 0.79 & 0.00 \\
\hline & TEOL & 111.39 & 1.00 & 111.39 & 17.17 & 0.00 & 0.42 \\
\hline & Treatment & 19.37 & 1.00 & 19.37 & 2.99 & 0.10 & 0.11 \\
\hline & Error & 155.69 & 24.00 & 6.49 & & & \\
\hline & \multicolumn{7}{|c|}{ a. RSquared $=.515($ Adjusted R Squared $=.474)$} \\
\hline
\end{tabular}

\section{Phase two}

Primary Endpoint: Pain Intensity

During phase two, the intervention group (crossed over from control) showed a mean present pain intensity of 2.94 , a mean worst pain of 4.56 and a mean least pain of 1.06. On the other hand, the control group (crossed over from intervention) showed a mean present pain intensity of 4.63, a mean worst pain of 7.09 and a mean least pain of 3.18. In table 5, a one way between subjects ANCOVA was conducted to determine a statistically significant effect of treatment on pain Intensity after controlling for initial intensity, present pain intensity (IPP) F (1, $26)=0.53, p=0.47$, worst pain (WP) $\mathrm{F}(1,24)=$ 2.83, $p=0.11$, least pain (LP) $\mathrm{F}(1,24)=2.32, p=$ 0.14 . 
Table 4: Tests of Between-Subject Effects of pain intensity of chronic pain patients with degenerative conditions at $\mathrm{UTH}$

\begin{tabular}{|c|c|c|c|c|c|c|c|}
\hline Variable & Source & $\begin{array}{l}\text { Type III } \\
\text { Sum of } \\
\text { Squares }\end{array}$ & $d f$ & $\begin{array}{l}\text { Mean } \\
\text { Square }\end{array}$ & $\mathrm{F}$ & Sig. & $\begin{array}{l}\text { Partial } \\
\text { Eta } \\
\text { Squared }\end{array}$ \\
\hline \multirow[t]{5}{*}{ Present Pain } & $\begin{array}{l}\text { Corrected } \\
\text { Model }\end{array}$ & $159.36^{\mathrm{a}}$ & 2.00 & 79.68 & 46.71 & 0.00 & 0.80 \\
\hline & Intercept & 7.48 & 1.00 & 7.48 & 4.38 & 0.05 & 0.15 \\
\hline & MPP & 140.54 & 1.00 & 140.54 & 82.39 & 0.000 & 0.77 \\
\hline & Treatment & 0.91 & 1.00 & 0.91 & 0.53 & 0.47 & 0.02 \\
\hline & Error & 40.94 & 24.00 & 1.71 & & & \\
\hline \multirow[t]{5}{*}{ Worst Pain } & $\begin{array}{l}\text { Corrected } \\
\text { Model }\end{array}$ & $238.40^{\mathrm{a}}$ & 2.00 & 119.20 & 101.74 & 0.00 & 0.89 \\
\hline & Intercept & 2.77 & 1.00 & 2.77 & 2.37 & 0.14 & 0.09 \\
\hline & MWP & 196.73 & 1.00 & 196.73 & 167.91 & 0.00 & 0.88 \\
\hline & Treatment & 3.32 & 1.00 & 3.32 & 2.83 & 0.11 & 0.11 \\
\hline & Error & 28.12 & 24.00 & 1.17 & & & \\
\hline \multirow[t]{5}{*}{ Least Pain } & $\begin{array}{l}\text { Corrected } \\
\text { Model }\end{array}$ & $164.56^{\mathrm{a}}$ & 2.00 & 82.28 & 43.60 & 0.00 & 0.78 \\
\hline & Intercept & 5.76 & 1.00 & 5.76 & 3.05 & 0.09 & 0.11 \\
\hline & MLP & 135.28 & 1.00 & 135.28 & 71.68 & 0.00 & 0.75 \\
\hline & Treatment & 4.39 & 1.00 & 4.39 & 2.32 & 0.14 & 0.09 \\
\hline & Error & 45.29 & 24.00 & 1.89 & & & \\
\hline
\end{tabular}

\section{Secondary Endpoints}

General Work

Results under general work showed a CBT mean of 3.25, [F $(1,24)=3.66, p=0.07]$, a control mean of 4.18 and initial general work $[\mathrm{F}$ $(1,24)=99.51, p=0.81]$

Mood

Results under mood interference showed a CBT mean of 0.38 , [F $(1,24)=0.41, p=0.53]$ and a control mean of 2.64 , with initial mood interference $[\mathrm{F}(1,24)=126.93, p=0.00]$

Normal work

Results under 'normal work' interference showed a CBT mean of 2.75, [F $(1,24)=2.80, p$ $=0.11]$ and a control mean of 4.00 with initial normal work $[\mathrm{F}(1,24)=45.45, p=0.00]$
Relations with people

Results under 'relations with other people' interference showed a CBT mean of 0.56 , $[\mathrm{F}(1,24)=0.80, p=0.38]$ with a control mean of 2.27 and $\operatorname{RWP}[\mathrm{F}(1,24)=531.89, p=0.00]$

Sleep

Results under sleep interference showed a CBT mean of 1.31, [F $(1,24)=1.78, p=0.20]$, a control mean of 2.36 and an initial sleep interference $[\mathrm{F}(1,24)=57.69, p=0.00]$

Enjoyment of Life

Results under 'enjoyment of life (EOL)' interference showed a CBT mean of 2.00, [F (1, $24)=3.24, p=0.08]$ with a control mean of 3.64 and an initial 'EOL' $[\mathrm{F}(1,24)=134.12, p=0.00]$ The ANCOVA results are shown in table 5. 
Nchawanangwa K, Kapenda C, Mwanza J, Chiluba BC,. A Crossover Design for Comparative Efficacy: A 28 Days-Randomized Clinical Trial of Cognitive Behavioral Therapy for Chronic Pain of Degenerative Conditions.

Table 5: Tests of between-subject effects of secondary outcomes of chronic pain patients with degenerative conditions at UTH

\begin{tabular}{|c|c|c|c|c|c|c|c|}
\hline Variable & Source & $\begin{array}{l}\text { Type III } \\
\text { Sum of } \\
\text { Squares }\end{array}$ & $2 f$ & $\begin{array}{l}\text { Mean } \\
\text { Square }\end{array}$ & $\mathrm{F}$ & Sig. & $\begin{array}{l}\text { Partial } \\
\text { Eta } \\
\text { Squared }\end{array}$ \\
\hline \multirow[t]{5}{*}{ General Work } & $\begin{array}{l}\text { Corrected } \\
\text { Model }\end{array}$ & $143.14^{4}$ & 2.00 & 71.57 & 51.80 & 0.00 & 0.81 \\
\hline & Intercapt & 0.23 & 1.00 & 0.23 & 0.17 & 0.69 & 0.01 \\
\hline & MGW & 137.48 & 1.00 & 137.48 & 99.51 & 0.00 & 0.81 \\
\hline & Treatment & 5.06 & 1.00 & 5.06 & 3.66 & 0.07 & 0.13 \\
\hline & Error & 33.16 & 24.00 & 1.38 & & & \\
\hline \multirow[t]{5}{*}{ Mood } & $\begin{array}{l}\text { Corrected } \\
\text { Model }\end{array}$ & $137.87^{2}$ & 2.00 & 68.93 & 83.71 & 0.00 & 0.88 \\
\hline & Intercept & 0.41 & 1.00 & 0.41 & 0.50 & 0.49 & 0.02 \\
\hline & MM & 104.53 & 1.00 & 104.53 & 126.93 & 0.00 & 0.84 \\
\hline & Treatment & 0.34 & 1.00 & 0.34 & 0.41 & 0.53 & 0.02 \\
\hline & Error & 19.76 & 24.00 & 0.82 & & & \\
\hline \multirow[t]{5}{*}{ Normal Work } & $\begin{array}{l}\text { Corrected } \\
\text { Model }\end{array}$ & $119.48^{x}$ & 2.00 & 59.74 & 24.85 & 0.00 & 0.67 \\
\hline & Intercapt & 0.08 & 1.00 & 0.08 & 0.03 & 0.86 & 0.00 \\
\hline & MNW & 109.29 & 1.00 & 109.29 & 45.45 & 0.00 & 0.65 \\
\hline & Treatment & 6.73 & 1.00 & 6.73 & 2.80 & 0.11 & 0.10 \\
\hline & Error & 57.71 & 24.00 & 2.40 & & & \\
\hline \multirow[t]{5}{*}{ Relations With People } & $\begin{array}{l}\text { Corrected } \\
\text { Model }\end{array}$ & $155.05^{2}$ & 2.00 & 77.53 & 303.24 & 0.00 & 0.96 \\
\hline & Intercapt & 0.01 & 1.00 & 0.01 & 0.06 & 0.82 & 0.00 \\
\hline & MRWP & 135.98 & 1.00 & 135.98 & 531.89 & 0.00 & 0.96 \\
\hline & Treatment & 0.21 & 1.00 & 0.21 & 0.80 & 0.38 & 0.03 \\
\hline & Error & 6.14 & 24.00 & 0.26 & & & \\
\hline \multirow[t]{5}{*}{ Sleep } & $\begin{array}{l}\text { Corrected } \\
\text { Model }\end{array}$ & $141.37^{2}$ & 2.00 & 70.69 & 30.39 & 0.00 & 0.72 \\
\hline & Intercept & 0.12 & 1.00 & 0.12 & 0.05 & 0.82 & 0.00 \\
\hline & MS & 134.17 & 1.00 & 134.17 & 57.69 & 0.00 & 0.71 \\
\hline & Treatment & 4.14 & 1.00 & 4.14 & 1.78 & 0.20 & 0.07 \\
\hline & Error & 55.82 & 24.00 & 2.33 & & & \\
\hline \multirow[t]{5}{*}{ Enjoyment of Life } & $\begin{array}{l}\text { Corrected } \\
\text { Model }\end{array}$ & $243.54^{4}$ & 2.00 & 121.77 & 72.23 & 0.00 & 0.86 \\
\hline & Intercept & 4.36 & 1.00 & 4.36 & 2.59 & 0.12 & 0.10 \\
\hline & MEOL & 226.09 & 1.00 & 226.09 & 134.12 & 0.00 & 0.85 \\
\hline & Treatment & 5.47 & 1.00 & 5.47 & 3.24 & 0.08 & 0.12 \\
\hline & Error & 40.46 & 24.00 & 1.69 & & & \\
\hline
\end{tabular}




\section{DISCUSSION}

The study results showed the outcomes of CBT as ranging from small to medium across various outcomes. Each outcome is analyzed and interpreted under different headings. The sociodemographic characteristics of the participants are discussed first. They are followed by the results concerning Pain intensity, general work and normal work interference, mood interference, relationship with people interference, sleep interference and finally enjoyment of life interference.

A total of twenty-seven participants were recruited from the University Teaching Hospital. All of the participants were above 18 years of age with the youngest being 25 years old and the oldest being 74 years old. Of the participants involved in the study, $63 \%$ were female and $37 \%$ were males. According to Fayaz et al chronic pain was more common in females than in males across all measured phenotypes [17]. Of the 27 participants, 19 suffered from conditions of the lumbar region, 3 from conditions of the cervical region, 2 from conditions of the thoracolumbar region, 2 from conditions of both the cervical and lumbar region and 1 from conditions of the knee. [6], showed CBT as being effective in a number of chronic pain conditions such as fibromyalgia, mixed chronic pain conditions, back pain, and arthritis and osteoarthritis

Chronic pain was seen to affect individuals over a variety of occupations and professions. The longest reported pain duration was 18 years while the least reported pain duration was 3 months. According to Majedi et al, the rate of inadequate pain management is between $25 \%$ and $82 \%$ in different populations and maybe due to the underestimation of pain intensity [18]. Pain killers were seen to be the most prevalent sort after treatment. Among the recruited participants, 59\% relied on painkillers only, 7.4\% relied on Physiotherapy only, 29.6\% relied both on pain killers and Physiotherapy and only $3.7 \%$ had never sort any treatment prior to the study.

Phase one of the study recorded small effects in the reduction of pain at present, worst and least levels. Phase two of the study also recorded small effects in the present pain intensity, worst experienced pain intensity and least experienced pain intensity.

Chronic pain resulting from degenerative conditions will start off being caused by physiological factors which results into psychosocial factors. Said psychosocial factors will result in a negative pain perception. This negative perception will magnify the intensity of the pain felt by the individual and ultimately result in reduced quality of life. CBT works on reducing the psychosocial factors and helping the patients learn to actively cope with the pain.

A study done by Lloyd showed that pain catastrophizing contributes to a more intense pain experience [19]. According to Quatarna et al and Edwards et al, patients tend to catastrophize their pain which in turn leads to an increase in their physical and psychosocial dysfunction $[20,21,22]$. Gatchel et al puts fear avoidance beliefs as another negative perception found in chronic pain patients. Gatchel states that the anticipated threat of intense pain will often result in the constant vigilance and monitoring of pain sensations which in turn will result in low intensity sensations of pain becoming unbearable [23]. This anticipation of increased pain sensations or of re-injury will stimulate avoidance behaviors which will result in the development of a vicious cycle. This will result into the avoidance of many activities which in turn will lead to inactivity and ultimately to disability. C.B.T interventions address a patient control perception and coping skills. Adaptive coping is a personality trait that is associated with better pain related functioning. Lindgreen et al and other studies state that C.B.T as a part of patient rehabilitation can encourage pain coping behavior by altering a patients' pain perception [24].

In phase one CBT had a significant but moderately small effect on the general work interference experienced. However, it had a medium effect on the normal work interference experienced. In phase two, CBT had a significant but moderately small effect on both the general work interference and the normal work interference experienced.

CBT having a moderately small effect on the general work interference in phase one and two and having a medium effect on normal work interference in phase one but a small effect in phase two may be as a result of the short follow up time. The study did however show that it does have an effect on the activity limitations that result from chronic pain. It is possible that in the long run it will further reduce the activity 
limitations that are experienced by individuals with chronic pain in degenerative conditions. The theory behind the reduction in interference can be explained by a break in the pain cycle that is achieved by not only pain treatment but a reduction in anxiety and catastrophizing. Chronic pain produces a cycle in which pain results in anxiety which in turn results in magnification of pain perception thus leading to avoidance of stimulus (in most cases- movement). Lack of movement in turn will interfere with an individuals' general and normal work and may result in disability.

Studies that looked at limitations in activities [25,6], recorded significant but small to moderate effects of CBT. These studies went on to support the efficacy of CBT compared to treatment as usual or wait-list conditions. Said studies also had follow-ups of 6-12 months. This goes against the theory of effects improving over time. When explaining the mechanism behind activity limitation, Quartana et al and Edwards et al recorded that activity limitations are said to be the outcome of pain catastrophizing which is one of the Cognitive distortions seen in patients with pain [20,21]. As catastrophizing is a negative Cognitive -affective response to anticipated or actual pain, it can be theorized that an individual will limit what they do in fear of pain onset or pain increment. Gatchel et al, supports this in that they record- the anticipation of increased pain sensations or of re-injury will stimulate avoidance behaviors which will result in the development of a vicious cycle [22]. In said cycle, the fear of pain or re-injury will contribute to the avoidance of many activities which in turn will lead to inactivity and ultimately to disability.

CBT produced a medium effect on mood interference in phase one but a small effect in phase two of the study. The medium effect produced can be argued to have been caused by a reduction in pain intensity or a reduction in pain related anxiety. However, as the research did not concentrate on pain-related anxiety, the theory route to be followed is one of pain intensity reduction. In this route, a patient experiencing chronic pain will be found to suffer some levels of mental distress and will also have cognitive distortions such as mental filtering and overgeneralization. This will make the person only concentrate on negative emotions hence the mood interference. According to Sharav and Benoliel, chronic pain has been associated with significant emotional distress and VachonPresseau et al, demonstrated the importance of the emotional brain (i.e., the corticolimbic system) in the prediction and amplification of chronic pain
$[23,24]$. Grohol and John address the aspect of mood interference due to chronic pain and explain it using the cognitive distortions, overgeneralizing and mental filtering [26]. In overgeneralizing, the individual assumes that all experiences are the same while in mental filtering an individual focus on the negative elements of the situation to the exclusion of the positive. This would lead the patient to only focus on the negative and ignore any positive. A Cochrane review by Williams, Eccleston, \& Morley, concluded that CBT has moderate effects on mood and post-treatment. They also recorded that by 6 - to 12 -month followup, the only significant effect was for mood [27].

Phase one recorded CBT as having no effect on how chronic pain interfered with how the patients related with others. Phase two on the other hand recorded a small effect in this aspect.

How individuals relate with others is dependent on aspects of their lives such as mood, sleep and how comfortable they are. In chronic pain, an individual will suffer from sleep disturbance which will leave them fatigued and irritable. Not only will this affect their mood but the lack of sleep will add onto it. This overall will lead to a change in social patterns and in some cases, withdrawal. CBT works by reducing sleep disturbance, improving mood and decreasing negative pain coping skills thus hitting the problem from various angles depending on the patient. A study done by Qaseem et al records individuals with sleep disturbance often experiencing fatigue, poor cognitive function, mood disturbance and distress which will interfere with behavioral, social, occupational, educational, academic, or other important areas of functioning [28].

For both phase one and phase two, CBT reported a moderately small effect size on the reduction of sleep interference in individuals experiencing chronic pain. This effect on sleep interference can be explained by the reduction in pain intensity. A reduction in an individual's pain levels leads to a reduction in sleep problems and an increase in sleep quality and length. An improvement in sleep will in turn result in further pain reduction. The cycle however plays out over a longer period of time and has factors such as anxiety and catastrophizing in play. Koffel et al in a meta-analysis of group CBT, demonstrated medium-to-large effect sizes for sleep onset latency, sleep efficiency, and wake after sleep onset [29]. According to Almoznino et al, there is a bidirectional relationship between poor sleep and pain, where pain leads to sleep difficulties that exacerbate the pain [30]. Carra et al, argues that the treatment of one (pain/sleep) can be helpful 
for the other (pain/ sleep) [31]. Vitiello et al, states that CBT strategies not only change sleep patterns, but may also improve pain in adults [32]. CBT has been found to be effective in the treatment of insomnia secondary to chronically painful medical conditions such as fibromyalgia, mixed chronic pain conditions, back pain, and arthritis and osteoarthritis $[33,23]$.

The study found that there was a small effect size on how CBT negated the effects of chronic pain and boosted the participant's enjoyment of life. Individuals with chronic pain reported that they could not perform pleasant activities as this always resulted in exacerbation of chronic pain. In some cases, the presence of pain even before the activities resulted in individuals fearing even trying to perform said pleasant activities. Pleasant activities are not only affected by chronic pain intensity but also by factors such as the amount and quality of sleep; and the amount of distress, catastrophizing, fear avoidance and overgeneralizing taking place.

The roundabout way in which chronic pain reduces an individuals' enjoyment of life can be seen in various studies. For example, O'Brien et al, records that depressive symptoms mediate poor sleep which Cardin et al, says interferes with routine functioning and affect patients' social lives thus according to Tage et al, (2013) collectively worsening the quality of life $[34,35,36]$. A meta-analysis by Hoffman, et al of 22 RCTs recorded psychological treatments of chronic back pain as having positive effects on the pain itself as well as pain-related interference with activities, health-related quality of life, and depression [37]. This can be summarized by stating that the impact of chronic pain is associated with substantial disability, distress and poor quality of life $[38,39,40,41,42,43]$.

\section{CONCLUSION}

As a physiotherapists' primary goal is pain reduction, it is important that said physiotherapist is able to understand the factors that affect the pain intensity. Pain intensity leads to activity and functional limitations of which combating them is the physiotherapists long term goal. It is therefore important to establish how a patient's emotions, beliefs and behaviors would lead to increased pain intensity and reduced activities and function. Cognitive Behavioral Therapy ensures the management of chronic pain addresses areas in which an individual is affected by pain and which in turn exacerbate the chronic pain. Physiotherapy therefore provides a more holistic approach when used in conjunction with CBT. At the time of study, there is no evidence that has been documented on the use of CBT strategies in the Physiotherapy management of chronic pain patients at the university teaching hospital.

\section{DECLARATION}

Author Contribution NK, BCC and CK developed study concepts. BCC designed and analyzed data in the study. JM contributed further conceptual meaning paradigms to the study. The manuscript was written by BCC and NK and all author went through the manuscript before submission.

Acknowledgement The authors would like to thank our patients at the department of physiotherapy who participated and consented to be part of this study. The authors also wish to thank 4th year physiotherapy students that participated in data collection of this study.

\section{Funding None}

Competing interests There were no competing interests from all authors in this study.

\section{REFERENCES}

1. Green L, Mcghie J. Assessment of acute and chronic pain, 2011; 12(1) Anesthesia and Intensive care Medicine, https://doi.org/10.1016/j.mpaic.2010.10.010

2. Henschke N, OsteloR.W, Van TulderM.W, VlaeyenJ.W, Morley S, Assendelft W.J, Main C.J, Behavioral treatment for chronic low-back pain, Cochrane Database Syst Rev 2010;7:CD002014.

3. Beck A, Rush J, Shaw B, Emery G, Cognitive Therapy of depression, 1979; New York: The Guilford Press

4. Martin B, In-Depth: Cognitive Behavioral Therapy, Psych Centre, 2016. www.psychcentral.com/lib/indepth-Cognitive -behavioral-therapy

5. Melzack R, Wall P. D, Pain mechanisms: A new theory, Science, 1965; $150,971-979$, doi:10.1126/science.150.3699.971

6. Ehde M.D, Dillworth M.T, Turner A.J, Cognitive Behavioral Therapy for Individuals with Chronic Pain Efficacy, Innovations, and Directions for Research. 2014; University of Washington

7. Somers T.J, Wren A.A, Shelby R.A, the context of pain in arthritis: self-efficacy for managing pain and other symptoms, Curr. Pain Headache Rep. 2012; 16, 502-508.

8. Koffel E.A, Koffel J.B, Gehrman P.R, A meta-analysis of group Cognitive Behavioral Therapy for insomnia, Sleep Med Rev 2014; 19: 6-16 
9. Bandura A, Social Foundations of Thought and Action: A Social Cognitive Theory, Englewood Cliffs 1986, Prentice Hall.

10. Turk D.C, Fillingim R.B, Ohrbach R, Patel K.V, Assessment of psychosocial and functional impact of chronic pain. J. Pain 2016; 17, T21-49.

11. French D.J, Holroyd K.A, Pinell C, Malinoski P.T, O'Donnell F, Hill K.R, Perceived self-efficacy and headache-related disability, Headache 2000; 40, 647-656.

12. Kalapurakkel S, Carpino E.A, Lebel A, Simons L.E, "Pain Can't stop me": examining pain self-efficacy and acceptance as resilience processes among youth with chronic headache, J. Pediatr. Psychol. 2014; 40, 926-933.

13. Costa L.C, Maher C.G, McAuley J.H, Hancock M.J, Smeets R.J, Self-efficacy is more important than fear of movement in mediating the relationship between pain and disability in chronic low back pain. Eur. J. Pain 2011; 15, 213-219.

14. Burns J.W, Nielson W.R, Jensen M.P, Heapy A, Czlapinski R, Kerns R.D, Specific and general therapeutic mechanisms in Cognitive Behavioral treatment of chronic pain, J. Consult. Clin. Psychol. 2015; 83, 1-11.

15. Turner J.A, Holtzman S, Mancl L, Mediators, moderators, and predictors of therapeutic change in Cognitive Behavioral Therapyfor chronic pain, Pain 2007; 127, 276286.

16. Brister H, Turner J.A, Aaron L.A, Mancl L, Self-efficacy is associated with pain, functioning, and coping in patients with chronic temporomandibular disorder pain, J. Orofac. Pain 2006; 20, 115-124.

17. Fayaz A, Croft P, Langford R.M, Donaldson L.J, Jones G.T, (2015), Prevalence of chronic pain in the UK: a systematic review and meta-analysis of population studies, 2015; (6) 6: http://dx.doi.org/10.1136/bmjopen-2015-010364

18. Vachon-Presseau E, Centeno MV, Ren W, The emotional brain as a predictor and amplifier of chronic pain, J Dent Res 2016; 95: 605-612.

19. Lloyd I, 2013, The Psychological Aspect of Pain,

20. Quartana P. J, Campbell C. M, Edwards R. R, Pain catastrophizing: A critical review, Expert Reviews Neurotherapy, 2009; 9(5), 745-758, doi:10.1586/ern.09.34

21. Edwards R. R, Cahalan C, Mensing G, Smith M, Haythornthwaite J. A, Pain, catastrophizing, and depression in the rheumatic diseases, Nature Reviews Rheumatology, 7(4), 216-224, doi:10.1038/nrrheum.2011. 2nrrheum.2011.2

22. Chiluba BC, Phiri J. Tackling Disability of Speech due to Stroke: Perspectives from Stroke Caregivers of the University Teaching Hospital in Zambia. Indonesian Journal of Disability Studies (IJDS).2019: Vol. 6(2): PP $215-222$.
23. Gatchel R.J, Neblett R, Kishino N, Ray C.T, FearAvoidance Beliefs and Chronic Pain, JOrthop Sports Phys Ther, PMID 2016: 26828236, DOI: 10.2519/jospt.2016.0601

24. Lindgreen $\mathrm{P}$, Rolving $\mathrm{N}$, Nielsen C.V, Lomborg K, Interdisciplinary Cognitive -Behavioral Therapyas part of lumbar spinal fusion surgery rehabilitation: experience of patients with chronic low back pain, OrthopNurs, 2016;35(4):238-47.

25. Gatchel R. J, Peng Y. B, Peters M. L, Fuchs P. N, Turk D. C, The biopsychosocial approach to chronic pain: Scientific advances and future directionsPsychological Bulletin, 2007; 133(4), 581-624, doi:10.1037/00332909.133.4.58.

26. Sharav Y, Benoliel R, Orofacial Pain and Headache, Quintessence: 2015Chicago.

27. Williams A. C, Eccleston, C, Morley S, Psychological therapies for the management of chronic pain (excluding headache) in adults, Cochrane Database of Systematic reviews, 2012(11), Article No. CD007407. doi:10.1002/14651858.CD007407.pub3.

28. Qaseem A, Kansagara D, Forciea M.A, Cooke M, Denberg T.D, Management of chronic insomnia disorder in adults: a clinical practice guideline from the American College of Physicians, Ann Intern Med 2006; 165: 125-133.

29. Keefe F.J, Somers T.J, Psychological approaches to understanding and treating arthritis pain, Nat, Rev Rheumato, 2010; 6: 210-216.

30. Almoznino G, Benoliel R, Sharav Y, Haviv Y, Sleep disorders and chronic craniofacial pain: characteristics and management possibilities, Sleep Med Rev 2016. doi: 10.1016/j.smrv.

31. Carra M.C, Bruni O, Huynh N, Topical review: sleep bruxism, headaches, and sleep-disordered breathing in children and adolescents, J Orofac Pain 2012; 26: 267-276.

32. Vitiello M.V, Rybarczyk B, Von Korff M, Stepanski E.J, Cognitive Behavioral Therapyfor insomnia improves sleep and decreases pain in older adults with co-morbid insomnia and osteoarthritis, J Clin Sleep Med 2009; 5: 355-362.

33. Roehrs T.A, Does effective management of sleep disorders improve pain symptoms? Drugs 69 2009(Suppl 2): 5-11.

34. O'Brien E.M, Waxenberg L.B, Atchison J.W, et al, Negative mood mediates the effect of poor sleep on pain among chronic pain patients, Clin J Pain, 2010;26(4):310319.

35. Cardin F, Ambrosio F, Amodio P, et al, Quality of life and depression in a cohort of female patients with chronic disease, BMC Surg. 2012;12 Suppl 1:S10.

36. Tage O, Taru K, Petteri K, et al, Anxiety and depression are independent predictors of quality of life of patients 
with chronic musculoskeletal pain, J Health Psych. 2013;18(2):167-175.

37. Hoffman B. M, Papas R. K, Chatkoff D. K, Kerns R. D, Meta-analysis of psychological interventions for chronic low back pain, Health Psychology, 2007; 26(1), 1-9. doi:10.1037/0278-6133.26.1.1

38. Smith B.H, Elliot A.M, Chambers W.A, et al, The impact of chronic pain in the community, Fam Pract, 2001;18(3):292-299.

39. Breivik H, Collett B, Ventafridda V, et al, Survey of chronic pain in Europe: prevalence, impact on daily life, and treatment, Eur J Pain, 2006;10(4):287-333.

40. Scholich S.L, Hallen D, Wittenberg, et al, The relationship between pain disability, quality of life and Cognitive -Behavioral factor in chronic back pain, Disabil Rehab. 2012;34(23):193-200.

41. Nchawanangwa K, Mwanza J, Kapenda C, Chiluba B.C, Efficacy of Cognitive Behavioral Therapy in Managing Chronic Pain of degenerative conditions: An Overview of Randomized Clinical Trials and Meta-Analysis Studies. J of Prev and Rehab Med, Vol. 2, No. 1, 2020, pp. 27-31. doi: $10.21617 /$ jprm2020.215.

42. Mpemba M, Shula HK, Chiluba BC. Stroke Disability and Physiotherapy Interventions: A Quantitative Evaluation of Physiotherapy Treatment Approaches' in Zambia. Indonesian Journal of Disability Studies (IJDS).2020: Vol. 7(1): pp. 92-100.

43. Nkandu-Munalula E, Simpamba-Mutuna M, Shula HK, Chisoso TL, Chiluba BC. Physiotherapy Intervention in Palliative Care for HIV Comorbidities: Can it be a Best Practice for Public Policy for Palliative Care in Zambia? Journal of Preventive and Rehabilitative Medicine, Vol. 2 No. 1, 2020, pp. 92-104. doi: 10.21617/jprm2020.224. 